\title{
The Application and Study of MATLAB in Electrical Engineering and Its Automation
}

\author{
Bowen Wang ${ }^{1, a}$
}

North China Electric Power University, Baoding 071000, China;

a2212334854@qq.com

Keywords: MATLAB; Electricity Engineering and Its Automation; Simulation; Teaching Design

\begin{abstract}
Electrical engineering courses are featured on combination of strong and weak electricity and take account into the specialized knowledge of the both. In the design of electricity tests and new products, it will significantly increase the complexity of test system and decrease teaching efficiency if adopting the real physical model design. While if simulating the real conditions by computers, it will achieve the effect that real tests don't have. Therefore, for the electrical engineering major, computer simulation plays a very important role. MATLAB has various tool bags and functional modules, including many corresponding electricity equipment models, which can achieve the analysis and design of motor speed control and electricity system, etc. And it has high-reliability simulation results and can fully mobilize the initiative of students. To analyze the application of MATLAB in electrical engineering and its automation, it gives a brief analysis of MATLAB first, and then analyzes the application of MATLAB in electrical engineering and its automation, expecting to offer some references for electrical engineering major teaching.
\end{abstract}

Electrical engineering and its automation has specialized knowledge of motor and its control, electricity system automation and manufacturing management, etc. It requires students to master such strong electricity fields as mathematics, computer, motor and high voltage, as well as weak electricity fields like operation control, research and development field. It has high speciality. In the specific teaching, although there are some differences in different universities' teaching focuses, yet all of them require the studies of electricity system and circuit and so on. Adopting traditional teaching modes will cost a lot and there may be test failures, so it's very bad for teaching. MATLAB software is one of the large aircraft application software. It has features of multidiscipline platform and involves a number of fields. It can be applied to Electrical engineering and automation speciality teaching. This article mainly analyzes the application of MATLAB software in Electrical engineering and its automation.

\section{General Introduction to MATLAB}

MATLAB is a kind of commercial teaching software produced by the U.S. MathWords Company. It is the combination of matrix and laboratory, meaning rectangular laboratory. It's one of the frequently used teaching software and mainly services to data visualization, data analysis and algorithm development, etc. The basic data unit of MATLAB software is matrix, similar to the directive expression frequently used in mathematics engineering. MATLAB software includes various functional tool bags software, such as numerical analysis, digital image processing, digital signal processing and demodulation and financial engineering, etc. This paper mainly analyzes Slimulink tool bag. Slimulink is a tool bag for model simulation and analysis, supporting linear and nonlinear system at the same time and can offer complete model base. It has visual and reloadable features and can significantly increase the reliability of simulation in the simulation tests. Due to its openness, MATLAB software allows users to independently design models they need, which is of great advantage in teaching.

Slimulink tool bags include subject models of power electronics, electric machinery and electrical engineering, which falls into 7 module bases: application base, measurement module base, discrete measurement module base, etc. Power module base includes DC voltage source, current source and AC power. Element module base includes transformer, switches and AC transformer, etc. 
Additional module base includes synchronization 6 pulse generator, clocks, discrete measurement module base and PID controller, etc. Measurement module base mainly includes current voltage measurement modules.

\section{Application of MATLAB in simulation of the Electrical Engineering and its Automation}

There are problems of various aspects existing in the experiment teaching activities of electrical engineering and its automation. For example, due to the establishment of digital platforms in most courses at present, students can accomplish the experiments via reading guide books even if they do not have a deep insight into the relevant knowledge, which leads to the separation of theory and practice, weakening the students' research ability. In addition, with the fast updating pace of electric appliance parts, the experimental conditions cannot meet the needs of some new-type experiments, large electric power experiments and so on.

The adoption of MATLAB software can enable the computer simulation design and virtual experiment to synchronously implement the design and experiment without the restrictions of components. Meanwhile, it will facilitate the adjustment and coverage of circuit parameters and realize the updating change of the electric appliance parts, which helps improve the comprehensive design and experiment ability of the students. The simulation is beneficial to cultivate students' thinking and innovation ability, ensure them to master experimental analysis methods on the basis of the grasp of software technology, and boost their study interest.

In the process of teaching activities of electrical engineering and its automation, theories concerning mainly include automatic control principles, modern control theories, power system theories and so on, which are excessively theoretical, and the adoption of traditional teaching methods can hardly achieve the ideal teaching effects. However, the adoption of MATLAB software will realize the application of simulation and enhance the figurativeness of theoretical knowledge and students' exploring desire.

The application of MATLAB software in the control system. In the professional learning process, the main content of the control system includes modern control theories, automatic control principles and so on. In the learning process of control principles, MATLAB software can be applied to the analysis and design of simulation experiments to get full knowledge of the system's working principles, control parameters and other contents. In the control system, the simulation analysis is mainly carried out by the Simulink system's qualitative experimental analysis to analyze the changes of the system performance along with different parameters and make improvements accordingly. For example, in the learning process of the analysis on spring-mass-damper mechanical displacement system, Chart 1 is a simulation model with a mass of $5 \mathrm{~kg}$, a spring damping factor $\mathrm{f}$ of 0.5 and an elasticity coefficient of 5 . Assuming that the initial speed of the object is 0 , under the condition that the external force $F(t)$ has changed, we analyze the displacement changes of the mass and further obtain knowledge of the system performance by the simulation results.

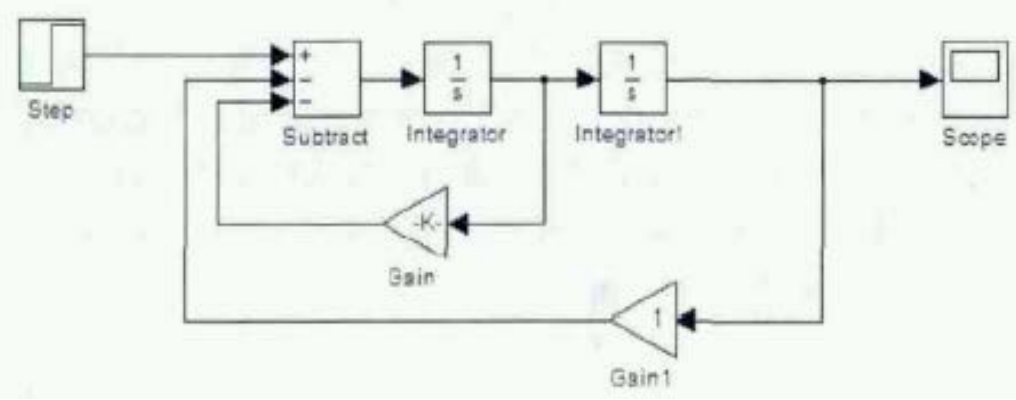

Figure 1 Spring-mass-damper mechanical displacement dynamic system simulation model 
The application of MATLAB software in the electric traction system. The electric traction system drives the operation of machines by motors and at the same time runs according to the relevant rules. In the electric drive system, the automatic control device is a major part. To control automatic machinery equipment, it is essential to find the corresponding regular changes, control the important physical parameters and the rotating speed of motors, and so on. The electric traction system includes mainly AC velocity modulation, pulse width modulation and so on, the simulation of which can all be realized by MATLAB software.

The application of MATLAB software in electricity system modeling. The electricity system belongs to the consumption and production system, including different parts like electricity generating, electricity distributing, electricity consuming and so on. Transient process, stable state running and so on need to be analyzed in electricity system, like Electricity System Tidal Current Energy Computation and short circuit failure computation and so on, which are all basic knowledge of electricity system learning. During learning, simulation modeling can be realized through the usage of MATLAB software. In practical work, staff can get the optimal parameters through simulation modeling by MATLAB software.

\section{The application of MATLAB in teaching of main curriculums}

Electrical engineering and its automation main curriculums include electricity and motor technology, automatic control system and so on. It is very complex in derivation of working principle, involving many ionization figures, waveform figures, formulas and so on. So it is of great difficulty in the adoption of traditional teaching. Application of MATLAB software can well resolve these problems. In the Siomulink part of MATLAB software, electricity system module includes DC asynchronous motor and synchronous magneto, which provides prodigious advantage for teaching.

Taking triphase bridge regulator circuit as an example, through modeling and parameter setting of Simulink and power system blockset, single-phase AC voltage source can be gained from the power system blockset and things like triphase voltage measurement unit and oscilloscope can be displayed clearly, and the simulation results are clear at a glance.

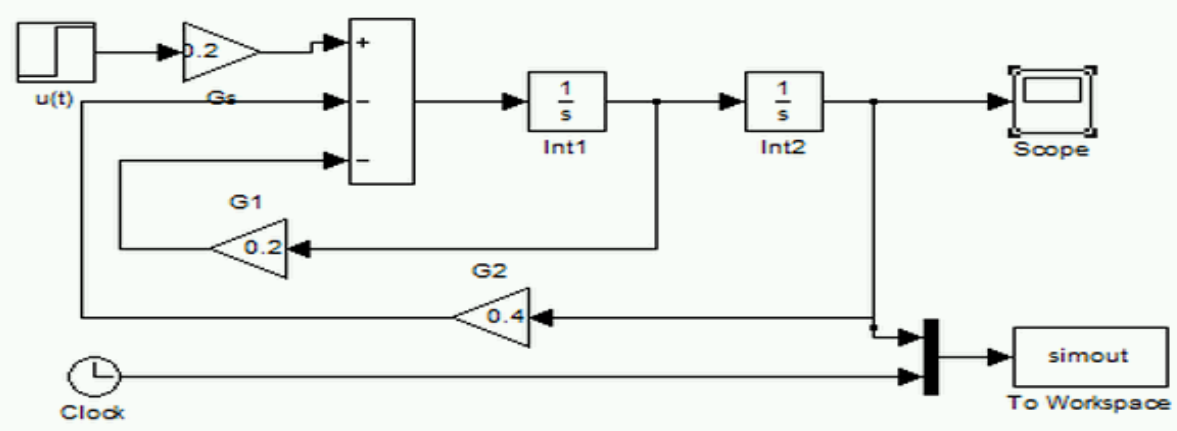

Figure 2 Simulation models and results of differential equation

Solve the differential equation by using integrator. In computation, second-order differential equation is required to be abstracted from realistic nature. Application of direct construction models by stages needs to be considered in mask, and differential equation is illustrated in figure 2 . After running, relevant simulation results can be gained.

The teaching of triphase bridge full controlled rectifier circuit. For example, in full controlled rectifier circuit, phase voltage electricity source, rectifier transformer output voltage are required to be $220 \mathrm{~V}$ and $100 \mathrm{~V}$ respectively, and observe the change of output voltage and current with different loads and trigger angles, as well the main subharmonic and AC side current waveform. Suppose that the resistor is $5 \mathrm{ohm}$, the peak of triphase electricity source voltage is 220 and the frequency is $50 \mathrm{~Hz}$, rectification ratio Nayaqi parameter primary winding connection and secondary winding connection are chosen to be D11 and Y respectively, of which line voltages are $380 \mathrm{~V}$ and $173 \mathrm{~V}$ respectively. If the demand is not strict, other transformers absorption numbers can remain 
unchanged. Primary winding connection and secondary winding connection of synchronous transformer are chosen to be D11 and Y respectively, of which line voltages are 380V and 150V respectively, and other parameters default to be unchanged. Simulation time is set to be $0.06 \mathrm{~s}$ and simulation experiment can begin after the setup of simulation parameters is done. Simulation results can be seen in figure 3. Rectifier output voltage is positive, and the change is consistent with the change of voltage waveform, but $\mathrm{Y}$ coordinate is different. If you change the control, the waveform change in different work situations can be observed.

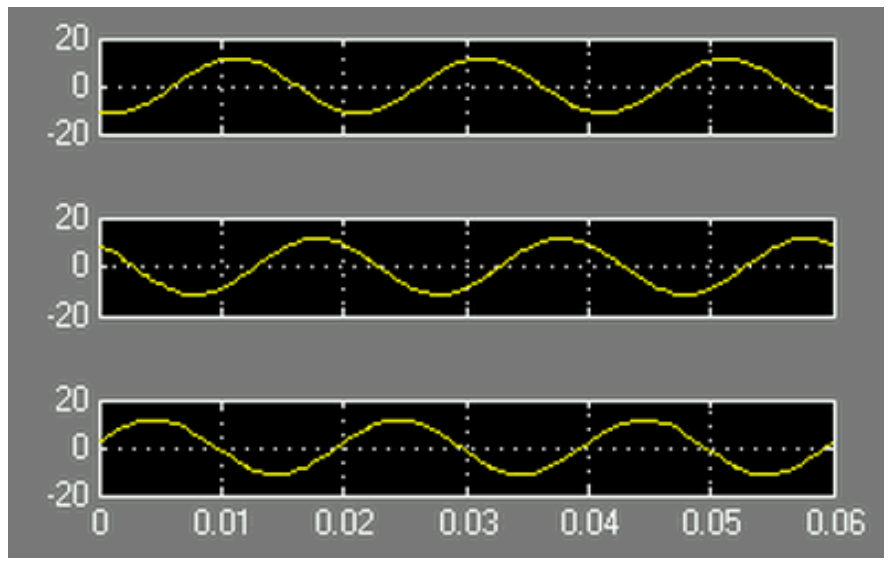

Figure 3 Changes when triphase current is input into the rectifier at 30 degrees

In the simulation model of resistance-inductance load (resistance value is set to the same of 5 ohm), the load PLC parameter should be modified. L value is set to $0.01 \mathrm{H}$, the trigger angle 60 degrees, the simulation time $0.16 \mathrm{~s}$, and starting the simulation model can get the output current and voltage value. During the current changes, there is a rising period followed by stability. It is applied in the current loop speed control of double closed-loop speed tuning. As shown in Figure 4, the simulation model is established in the integrated environment. After $0.3 \mathrm{~s}$, the unit step response signal is input, and the PID controller module is required to be put in as shown in the figure.

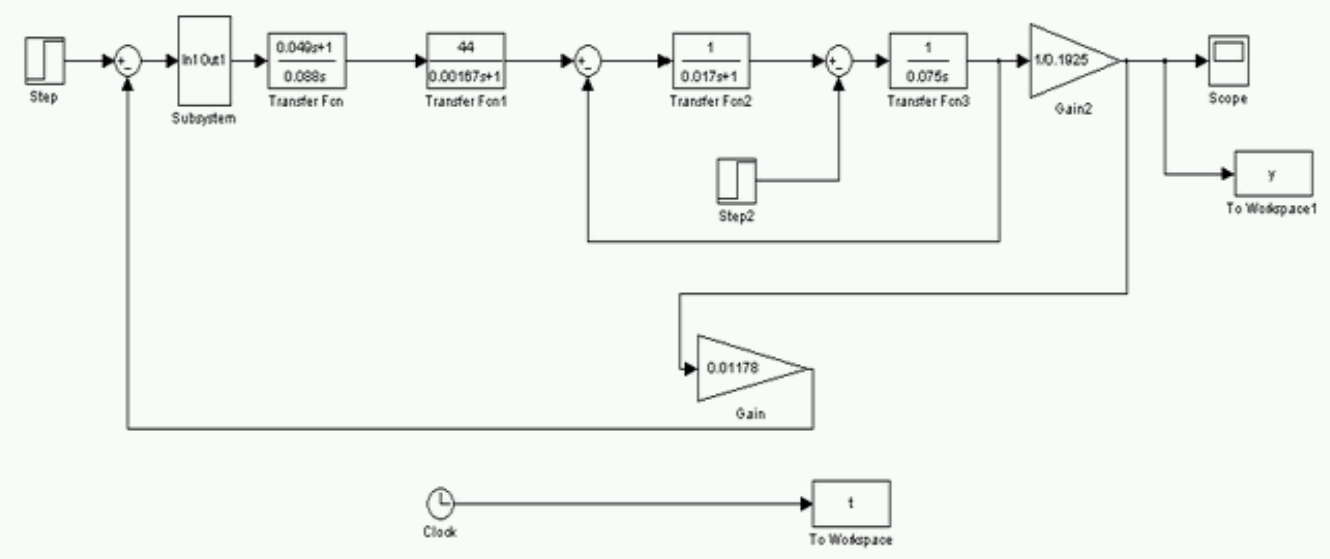

Figure 4 Double-loop speed controller applying the PID adjuster

\section{Application of MATLAB in Graduation Design}

In the graduation design of electrical engineering and its automation, there is a lot of professional knowledge, and the update rate is very fast. In the traditional teaching, priorities were focused on the electrical design or protective relaying, but little attention was paid to the popular electric power telecommunication and fault diagnosis. Moreover, in the traditional teaching, the literature search was very difficult, but the adoption of MATLAB software can be very useful to make up for the shortage of traditional teaching. 
In the process of graduation design, the students are very likely to encounter quite complex simulation programs. In the power system modeling and simulation of the graduation design, MATLAB software provides the students with various components needed by electrical major, so they can build a variety of power system models, then observe the adopted changing models, and verify the experimental results. As shown in Figure 5, MATLAB software is used to build a model of Electricity System Tidal Current Energy Computation. The data of node flow analysis could be obtained through the simulation, and results could be got through comparison with the design flow calculation results. Moreover, the analysis of establishing the model could offer help to the design of power system simulation interface.

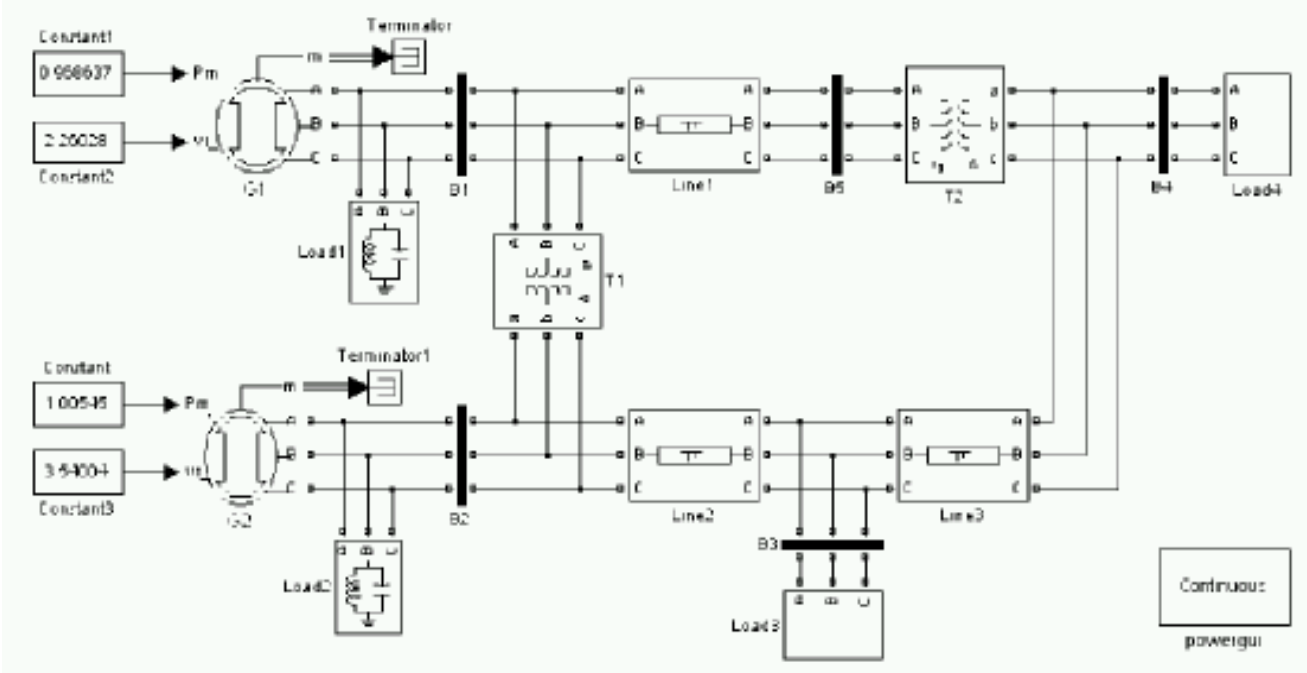

Figure 5 Simulation Model of Electricity System Tidal Current Energy Computation

Application of MATLAB in electric parameter acquisition. In the graduation design, electrical parameters involved include power, current and phase change, and these parameters could be implemented through the model. With the help of FFT analysis in the MATLAB software, the result could be obtained. The graphic editor in the MATLAB software could provide the corresponding observation modules for designers. For instance, in the oscilloscope module, they can clearly see the simulation results and waveform at the point of observation and analysis the parameter values at any time, and in the display module, they can directly read the current value and voltage value on every power line. The most typical example is the data reading of stable electric power parameters in the electric power system. In the neutral ungrounded system, the different phases on the line could be seen. With reasonable judgment, these obtained parameters can provide the basis for the judgment of the fault lines.

The use of GUI graphic interface design of MATLAB software can be of great help to electrical simulation interface design. Here graphical user interfaces mainly include window, cursor, menu, etc. Users can import and export correlation parameters through keyboard and use mouse to activate these parameters to complete the calculation of relevant data or waveform drawing, etc. The graphic interface design of MATLAB software is mainly implemented through image objects manipulation. Therefore, users need to be clear about the properties and characteristics of various graphic objects when using MATLAB software, which can fully train designer's design capability. For example, in the MATLAB simulation experiment of Electricity System Tidal Current Energy Computation, model and function files of GUI kernel were integrated, and sub-interface could be accessed by clicking corresponding buttons. In the mutually exclusive design of various menu bars on the main interface, mutually exclusive programs need to be added to realize this operation, for example: set(andles. radicbutton2, "value", 0); set(andles. radicbutton4, "value", 0); the returned value of unchecked and clicked buttons are set at 0 and 1 respectively to realize mutually exclusive function, and the programming capability and the capability to build an electrical model were also tested. 


\section{Conclusion}

In conclusion, this paper analyzes the application of MATLAB software in electrical engineering and its automation from different aspects. The process of teaching activities of electrical engineering and its automation involves knowledge of various subjects. In the teaching, students are required to master research methods of electrical engineering and its automation major on the basis of mastering basic theory, so as to enhance ability in practice. In simulation, the use of MATLAB software is conducive to involve teaching categories to achieve the aim of optimizing resource allocation as well as increasing students' interests and enthusiasm in participation.

\section{Reference}

[1] Zhu Junnan. Discussion on the Value of MATLAB Simulation Application in the Electrical Engineering and Its Automation [J]. Silicon Valley, 2014, 05:189-190.

[2] Zhang Lili, Wu Yan. The Simulation Application of MATLAB in the Electrical Engineering and Its Automation [J]. Computer CD Software and Application, 2012, 16:63-64.

[3] Xu Yubao, Zhang Xiaodong, Fang Jie, Yang Ting. Research and Reform of Practice Curriculum System of the Electrical Engineering and Its Automation [J]. China Electric Power Education, 2014, 31:46-47.

[4] Liu Duyu, Shao Shiquan. Discussion on the Teaching of Matlab in the Electrical Engineering and Its Automation [J]. Academic Journal of Southwest University for Nationalities (Natural Science Edition), 2011, S1:38-40.

[5] Su Liangyu, Zhao Zhongbiao, Shi Luhuan. Research on the Application of MATLAB in the process of teaching activities of electrical engineering and Its Automation [J]. Scientific and Technological Information, 2011, 18:11.

6] Li Xiao, Liu Tianye. Research on Engineering Talents Cultivation of the Electrical Engineering and Its Automation [J]. Academic Journal of North University of China (Social Science Edition), 2007, 01:83-86.

[7] Wei Liming, Han Chenghao, Wang Congze. Exploration on Comprehensive Teaching Reform of Applied Talents Cultivation of the Electrical Engineering and Its Automation [J]. Academic Journal of Jilin Jianzhu University, 2013, 01:91-93. 